\title{
Impact of the COVID-19 pandemic on surgery for thyroid cancer in Italy: nationwide retrospective study
}

\author{
F. Medas (ID 1,*, G. L. Ansaldo ${ }^{2}$, N. Avenia ${ }^{3}$, G. Basili ${ }^{4}$, M. Bononi ${ }^{5}$, A. Bove $^{6}$, P. Carcoforo ${ }^{7}$, A. Casaril ${ }^{8}$, G. Cavallaro (iD ${ }^{5}$, G. Conzo ${ }^{9}$, \\ L. De Pasquale ${ }^{10}$, P. Del Rio ${ }^{11}$, G. Dionigi ${ }^{12}$, C. Dobrinja ${ }^{13}$, G. Docimo ${ }^{14}$, G. Graceffa ${ }^{15}$, M. Iacobone (ID ${ }^{16}, N^{2}$ Innaro ${ }^{17}$, C. P. Lombardi ${ }^{18}$, \\ G. Novelli ${ }^{19}$, N. Palestini ${ }^{20}$, F. Pedicini ${ }^{21}$, G. Perigli ${ }^{22}$, A. Pezzolla ${ }^{23}$, L. Pezzullo ${ }^{24}$, G. Scerrino ${ }^{25}$, S. Spiezia ${ }^{26}$, M. Testini $^{27}$ and \\ P. G. Calò ; on behalf of the Italian Society of Endocrine Surgery (SIUEC) Collaborative Group
}

\author{
${ }^{1}$ Department of Surgical Sciences, University of Cagliari, Cagliari, Italy \\ ${ }^{2}$ Endocrine Surgery Unit, Ospedale Policlinico San Martino, Genoa, Italy \\ ${ }^{3}$ General Surgery and Surgical Specialties Unit, Santa Maria University Hospital Terni and University of Perugia, Medical School, Terni, Italy \\ ${ }^{4}$ General Surgery Department, Endocrine Surgery Unit, Azienda USL Toscana Nord-Ovest, Pontedera, Italy \\ ${ }^{5}$ Department of Surgery, Pietro Valdoni, Sapienza University of Rome, Rome, Italy \\ ${ }^{6}$ Department of Medicine, Dentistry and Biotechnology, University 'G. D'Annunzio', Chieti, Italy \\ ${ }^{7}$ Department of Surgery, Unit of General Surgery, University Hospital of Ferrara and University of Ferrara, Ferrara, Italy \\ ${ }^{8}$ Endocrine Surgery Unit, Pederzoli Hospital, Peschiera del Garda, Italy \\ ${ }^{9}$ Division of General and Oncological Surgery, Department of Translational Medical Sciences, University of Campania 'Luigi Vanvitelli', Naples, Italy \\ ${ }^{10}$ Endocrine Surgery, ASST Santi Paolo e Carlo University of Milan, Milan, Italy \\ ${ }^{11}$ General Surgery Unit, Parma University Hospital, Parma, Italy \\ ${ }^{12}$ Division for Minimally Invasive and Endocrine Surgery, University of Messina, Messina, Italy \\ ${ }^{13}$ Department of Medicine, Surgery and Health Sciences, Azienda Sanitaria Universitaria Giuliano Isontina, Trieste, Italy \\ ${ }^{14}$ Division of Thyroid Surgery, Department of Medical and Advanced Surgical Sciences, University of Campania 'Luigi Vanvitelli', Naples, Italy \\ ${ }^{15}$ Department of Surgical, Oncological and Stomatological Sciences, University of Palermo, Palermo, Italy \\ ${ }^{16}$ Endocrine Surgery Unit, Department of Surgery, Oncology and Gastroenterology, University of Padua, Padua, Italy \\ ${ }^{17}$ Unit of Endocrine Surgery, AOU Mater Domini, Catanzaro, Italy \\ ${ }^{18}$ Division of Endocrine Surgery, Department of Gastroenterological, Endocrine-Metabolic and Nephro-Urologic Sciences, Fondazione Policlinico Universitario \\ Agostino Gemelli IRCCS, Rome, Italy \\ ${ }^{19}$ Endocrine Surgery Unit, Department of Oncological-Mininvasive Surgery, Niguarda Hospital, Milan, Italy \\ ${ }^{20} \mathrm{Head}$ and Neck Oncological Surgery, Candiolo Cancer Institute, Candiolo, Italy \\ ${ }^{21}$ Thyroid Endocrine Surgery, Sant'Eugenio Hospital, Rome, Italy \\ ${ }^{22}$ Endocrine Surgery Unit, University of Florence, Florence, Italy \\ ${ }^{23}$ Division of Videolaparoscopic Surgery, Department of Emergency and Organ Transplantation, University of Bari 'A. Moro', Bari, Italy \\ ${ }^{24}$ Thyroid Surgery Unit, INT IRCSS Fondazione Pascale, Naples, Italy \\ ${ }^{25}$ Department of Surgical Oncology and Oral Sciences, Unit of General and Emergency Surgery, University of Palermo, Palermo, Italy \\ ${ }^{26}$ Endocine and Ultrasound-Guided Surgery Operative Unit, Ospedale del Mare, Naples, Italy \\ ${ }^{27}$ Unit of Academic General Surgery 'V. Bonomo', Department of Biomedical Sciences and Human Oncology, University of Bari 'A. Moro', Bari, Italy \\ *Correspondence to: Department of Surgical Sciences, University of Cagliari, Via Università 40, 09124 Cagliari, Italy (e-mail: fabiomedas@unica.it) \\ Members of the SIUEC Collaborative Group are co-authors of this study and are listed under the heading Collaborators
}

\section{Dear Editor}

The COVID-19 pandemic has presented a serious burden to public health. Italy has been affected heavily, with nearly 2 million confirmed cases and almost 70000 deaths. Globally, healthcare authorities have restricted medical care to emergency procedures, postponing elective surgical activity ${ }^{1-5}$. To evaluate the impact of restrictions on surgical activity for thyroid cancers in Italy, the Italian Society of Endocrine Surgery group and the Oncoteam of the Italian Society of Oncological Surgery undertook a retrospective analysis, comparing activity during the first wave of the COVID-19 pandemic (from 9 March 2020 to 31 August 2020) with activity during the same period of the previous year (control group). The study was registered at ClinicalTrials.gov (NCT04635813).

For this study, aggregated data were collected from 28 Italian surgical units, including patients who had undergone thyroidectomy with a diagnosis of thyroid carcinoma during the reference intervals. The main outcome of this study was the reduction in surgical activity for thyroid cancer. Features of the neoplasms diagnosed, including tumour size and incidence of lymph node metastases, extrathyroidal extension and multicentricity, were also analysed, to identify any significant differences between the two groups.

A total of 1570 patients with thyroid cancer were included in the study; 662 patients with thyroid carcinoma had surgery during the COVID-19 pandemic, and 908 during the same period in the previous year. This represented a 27.1 per cent reduction in the number of operations (Fig. 1). The mean(s.d.) number of patients operated on per unit was 21.6(24.5) in 2020 and 23.6(21.6) in 2019 ( $P=0.158)$. Mean nodule size tended to be larger in the group that had surgery during the pandemic (18.4(13.5) versus 17.2(12.6) $\mathrm{mm} ; \mathrm{P}=0.071)$. The incidence of lymph node metastases (21.5 per cent in 2020 versus 18.1 per cent in 2019; 


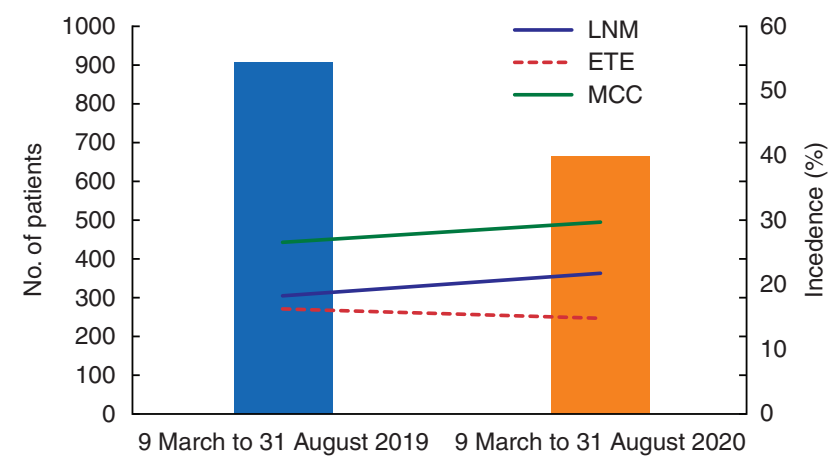

Fig. 1 Number of patients operated upon during 2019 and 2020, and incidence of lymph node metastasis, extrthyroidal extension and multicentric carcinoma.

Bars indicate number of patients and lines the incidence of lymph node metastasis (LNM), extrathyroidal extension (ETE) and multicentric carcinoma (MCC)

$P=0.107)$, extrathyroidal extension (14.7 versus 16.0 per cent; $P=0.520$ ), and multicentricity (29.5 versus 26.4 per cent; $P=0.205$ ) did not differ between the two groups.

Overall, there was a reduction in thyroidectomies for thyroid malignancies during the COVID-19 pandemic. This reduction was, however, not significant, probably because elective surgery was reserved for the most aggressive thyroid malignancies rather than uncertain or benign thyroid disease during the most difficult phases at the start of the pandemic. With the weakening of the pandemic in the summer months, surgical activity was gradually restored. A greater proportion of aggressive tumours was not observed during the COVID-19 pandemic. Nevertheless, this aspect should be monitored carefully because the delay in screening programmes and planned examinations could lead to an increase in missed diagnoses in the future, with an increased number of aggressive tumours.

Considering the new escalation of the pandemic while this report was being written, healthcare organizations are urged to ensure prompt treatment for thyroid malignancies, including patients with suspicious nodules. The authors recommend not delaying screening programmes and planned visits, which could be moved to non-hospital settings.

\section{Collaborators}

G. Anedda (University of Cagliari, Cagliari), G. Antonelli (Ospedale del Mare, Naples), G. Arrigoni (Candiolo Cancer Institute, Candiolo), B. Badii (University of Florence, Florence), E. Bonati (Parma University Hospital, Parma), M. Boniardi (Niguarda Hospital, Milan), A. M. Bulfamante (ASST Santi Paolo e Carlo University of Milan, Milan), V. Candalise (Azienda USL Toscana Nord-Ovest, Pontedera), A. Cangiano (University of Campania 'Luigi Vanvitelli', Naples), G. L. Canu (University of Cagliari, Cagliari), F. Cappellacci (University of Cagliari, Cagliari), A. Caracciolo (Candiolo Cancer Institute, Candiolo), E. Caruso (University of Messina, Messina), E. Casal Ide (University of Padua, Padua), A. Chiappini (Sapienza University, Rome), M. G. Chiofalo (INT IRCSS Fondazione Pascale, Naples), C. Cipolla (University of Palermo, Palermo), L. Costigliola (INT IRCSS Fondazione Pascale, Naples), F. Cozzani (Parma University Hospital, Parma), A. Crocco (INT IRCSS Fondazione Pascale, Naples), D. Crocetti (Sapienza University, Rome), N. de Manzini (Azienda Sanitaria Universitaria Giuliano Isontina, Trieste), A. Di Gioia (University 'G. D’Annunzio', Chieti), V. Di Resta (University
'G. D’Annunzio', Chieti), R. Eramo (Azienda Sanitaria Universitaria Giuliano Isontina, Trieste), E. Erdas (University of Cagliari, Cagliari), S. Ferriolo (University of Palermo, Palermo), M. Filardo (University of Campania 'Luigi Vanvitelli', Naples), M. Filograna Pignatelli (University of Campania 'Luigi Vanvitelli', Naples), R. Gervasi (AOU Mater Domini, Catanzaro), F. Giudici (University of Florence, Florence), L. Gordini (Fondazione Policlinico Universitario Agostino Gemelli IRCCS, Rome), A. Gurrado (University of Bari, Bari), H. Impellizzeri (Pederzoli Hospital, Peschiera del Garda), M. Inama (Pederzoli Hospital, Peschiera del Garda), M. Koleva Radica (University of Ferrara, Ferrara), R. Laforgia (University of Bari, Bari), S. Lattarulo (University of Bari, Bari), T. Loderer (Parma University Hospital, Parma), R. Lucchini (University of Perugia, Terni), F. Mascioli (Sapienza University Rome), R. Marcellinaro (Sant'Eugenio Hospital, Rome), R. Menditto (Sant'Eugenio Hospital, Rome), G. Melfa (University of Palermo, Palermo), M. Minuto (Ospedale Policlinico San Martino, Genoa), C. Misso (Ospedale del Mare, Naples), C. Offi (Ospedale del Mare, Naples), G. Orlando (University of Palermo, Palermo), P. Ossola (Sapienza University, Rome), C. Pagetta (University of Padua, Padua), A. Pasculli (University of Bari, Bari), R. Patrone (University of Campania 'Luigi Vanvitelli', Naples), I. Pauna (Niguarda Hospital, Milan), F. Pennetti Pennella (University of Perugia, Terni), D. Pietrasanta (Azienda USL Toscana Nord-Ovest, Pontedera), A. Pino (University of Messina, Messina), V. L. Pinto (University of Bari, Bari), S. Piras (University of Cagliari, Cagliari), A. Polistena (Sapienza University, Rome), M. Portinari (University of Ferrara, Ferrara), S. Reina (Ospedale Policlinico San Martino, Genoa), G. Rotolo (University of Palermo, Palermo), G. Russo (Sapienza University, Rome), E. Scalise (University of Campania 'Luigi Vanvitelli', Naples), L. I. Sgaramella (University of Bari, Bari), M. G. Sibilla (University of Ferrara, Ferrara), S. Spinelli (University of Bari, Bari), D. Spoletini (Sant'Eugenio Hospital, Rome), L. S. Curto (AOU Mater Domini, Catanzaro), M. Tascone (Candiolo Cancer Institute, Candiolo), F. Torresan (University of Padua, Padua), E. Varaldo (Ospedale Policlinico San Martino, Genoa), E. Viviani (Pederzoli Hospital, Peschiera del Garda), A. Zucca (Azienda Sanitaria Universitaria Giuliano Isontina, Trieste).

Disclosure. The authors declare no conflict of interest.

\section{References}

1. Søreide K, Hallet J, Matthews JB, Schnitzbauer AA, Line PD, Lai PBS et al. Immediate and long-term impact of the COVID-19 pandemic on delivery of surgical services. Br J Surg 2020;107: 1250-1261.

2. Valmasoni M, Pierobon ES, Capovilla G, Piangerelli A, Moletta L, Costantini $\mathrm{M}$ et al. Providing surgery for cancer during the COVID19 pandemic: experience of a northern Italian referral centre. $\mathrm{Br} J$ Surg 2020;107:e326-e327

3. Spinelli A, Pellino G. COVID-19 pandemic: perspectives on an unfolding crisis. BrJ Surg 2020; 107:785-787

4. Indini A, Aschele C, Cavanna L, Clerico M, Daniele B, Fiorentini G et al. Reorganisation of medical oncology departments during the novel coronavirus disease-19 pandemic: a nationwide Italian survey. Eur J Cancer 2020;132:17-23

5. Di Marzo F, Sartelli M, Cennamo R, Toccafondi G, Coccolini F, La Torre $G$ et al. Recommendations for general surgery activities in a pandemic scenario (SARS-CoV-2). Br J Surg 2020;107: 1104-1106 